\title{
Gait Recognition Based on Modified Phase Only Correlation
}

\author{
Imad Rida ${ }^{1}$, Ahmed Bouridane ${ }^{2}$, Samer Al Kork ${ }^{3}$, and François Bremond ${ }^{4}$ \\ 1 LITIS EA 4108 - INSA de Rouen, St Etienne du Rouvray, France \\ ${ }^{2}$ Department of Computer Science and Digital Technologies \\ Northumbria University, Newcastle, UK \\ 3 Signal Processing and Machine Learning Lab, ESPCI-Paris-Tech, Paris, France \\ 4 INRIA Sophia Antipolis, STARS team Sophia Antipolis, France \\ imad.rida@insa-rouen.fr, ahmed.bouridane@northumbria.ac.uk, \\ samer_alkork@hotmail.com, Francois.Bremond@inria.fr
}

\begin{abstract}
Clothing, carrying conditions, and other intra-class variations, also referred as "covariates", affect the performance of gait recognition systems. This paper proposes a supervised feature extraction method which is able to select relevant features for human recognition to mitigates the impact of covariates and hence improve the recognition performance. The proposed method is evaluated using CASIA Gait Database (Dataset B) and the experimental results suggest that our method yields attractive results.
\end{abstract}

Keywords: Gait, phase only correlation, feature selection.

\section{Introduction}

Technology has invaded our lives as never before and the effectiveness of current security systems has become increasingly important. The development of automatic personal identification systems has increased in recent years and worldwide effort has been devoted to broaden and enhance personal identification systems. In particular, biometric recognition has become an area of particular interest and is used in numerous applications. Biometric recognition aims to identify individuals using unique, reliable and stable physiological and/or behavioral characteristics such as fingerprint, palmprint, face, gait, etc . Gait recognition consists on discriminating among people by the way or manner they walk. Gait as a biometric trait can be seen as advantageous over other forms of biometric identification techniques for the following reasons: (i) The gait of a person walking can be extracted and analysed from distance without any contact with sensor and (ii) The images used in Gait recognition can be easily provided by low-resolution, video-surveillance cameras. The major challenges of gait recognition are due to the effect of various covariates which are due to the presence of shadows, clothing variations and carrying conditions (backpack, briefcase, handbag, etc). From a technical point of view segmentation and the view dependency are further causes of the gait recognition errors. 
The aim of this paper is to mitigate the effect of the covariates to improve the recognition rate. In our approach we propose a wrapper feature selection algorithm by exploiting Modified Phase-Only Correlation (MPOC) method. MPOC is a modified Phase-Only Correlation (POC) using a band-pass type spectral weighting function in order to achieve better performances. MPOC is an efficient method that has been successfully applied to partial shoeprints classification [3]. It is effective and efficient method to match images with low texture feature.

The rest of this paper is organized as follows: Sect. 2 summarizes the previous works. Sect. 3 gives the theoretical description of the proposed method. Sect. 4 presents the experimental results. Sect. 5 offers our conclusion.

\section{Related Works}

Gait recognition techniques can be classified into two main categories: model based and model free methods. Model based approaches [9] use the estimation over time of the parameters of the body models for recognition. This process is usually computationally intensive since we need to model and track the subjects body and we also require high resolution images. Due to these limitations in recent times many model free approaches have been proposed. In a model free approach, the representative features of gait are derived from the moving shape of the subject. In this approach, the gait signature is derived from spatiotemporal patterns of a walking person [8], optical flow 6], variation of area within particular region [2], Gait Energy Image (GEI) 4] which is a spatiotemporal gait representation, Gait Moment Image (GMI) [7. The major disadvantage of this method relates to effect of covariates (clothes, carrying conditions). Recently some works have been proposed to make the model free approach robust against covariates. Bashir et al. introduced Gait Entropy Image (GEnI) [1] to perform automatic feature selection and flow fields [1].

\section{$3 \quad$ Methodology}

There exist a number of features for use in gait recognition, each type having advantages and disadvantages. The best feature representation should make a compromise between the computational cost and the recognition performance. Recently Gait Energy Image (GEI) has been widely used as a descriptor, which is simple and easy to compute.

A number of gait recognition methods using GEI have been proposed. Yu et al used template matching for the whole GEI without any feature selection [10]. Han and Bhanu suggested Canonical Discriminant Analysis (CDA) 4] corresponding to Principal Component Analysis (PCA) followed by a Multiple Discriminant Analysis (MDA). Due to the variance effects, Bashir et al [1] introduced Gait Entropy Image (GEI) to select features which are not affected by the covariant conditions. The idea is to take the features having a high entropy corresponding to the dynamic parts of the body to enhance the recognition. 


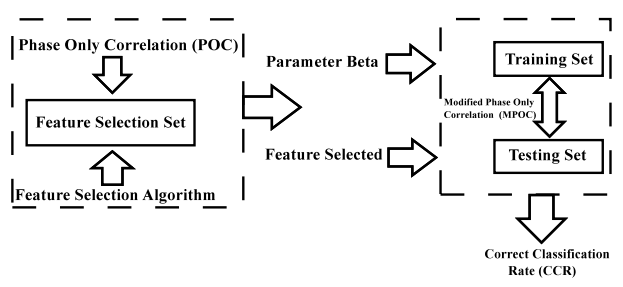

Fig. 1. Scheme representing modules of our method

In this paper, we propose to combine GEI features which are an efficient representation of the gait and MPOC algorithm of matching which has a good performance in low resolution images [3. We also propose a wrapper feature selection algorithm to select relevant features. As is shown in Fig. 1] our method is divided into 2 modules: the first one is used to select feature subset and the parameter of the spectral weighting function and the second one computes the final Correct Classification Rate (CCR).

\subsection{Gait Energy Image}

The human walk is considered as a cyclic activity where the motion repeats at a constant frequency. Gait Energy Image is a representation of human walk using a single grayscale image obtained by averaging the silhouettes extracted over a complete gait cycle 4]. GEI is computed using this equation

$$
G(x, y)=\frac{1}{N} \sum_{t=1}^{N} B(x, y, t)
$$

Where $N$ is the number of the frames within complete gait cycle, $B$ is a silhouette image, $x$ and $y$ are the coordinate of the image and $t$ is frame number in the cycle. Pixels with low intensity correspond to the dynamic parts of the body(lower part of the body) and this part of the GEI is very useful for the recognition and is not affected by the covariates as carrying and clothing conditions. Pixels with high intensity correspond to the static parts of the body (top part of the body), and this part contains the body shape information which can be useful for identification but it can be affected by the covariate conditions [1.

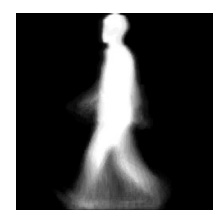

(a)

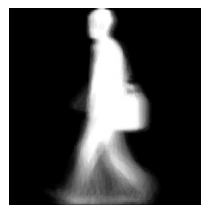

(b)

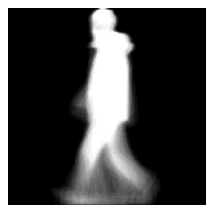

(c)

Fig. 2. Gait energy image of an individual under different conditions 


\subsection{Phase Only Correlation}

Since in the Fourier domain, the phase information captures (preserves) more features of the patterns than the magnitudes, phase-based image matching can be very attractive. This technique was already successfully used in biometric applications such as palmprint [5. This section shows the definition and concept of the Phase-Only Correlation function on GEI.

Consider two images $f(n, m)$ and $g(n, m)$ each having a size of $N_{1} \times N_{2}$ where $N_{1}=2 N+1$ and $N_{2}=2 M+1$ so that the index range of $n$ and $m$ are $-N \ldots . . N$ and $-M \ldots . . M$, respectively. Let $F(u, v)$ and $G(u, v)$ denote 2D DFTs of two images which can be written as follows:

$$
\begin{aligned}
F(u, v) & =\sum_{n=-N}^{N} \sum_{m=-M}^{M} f(n, m) e^{\frac{-2 j u n \pi}{N_{1}}} e^{\frac{-2 j v m \pi}{N_{2}}} \\
& =A_{F}(u, v) e^{j \theta_{F}(u, v)} \\
G(u, v) & =\sum_{n=-N}^{N} \sum_{m=-M}^{M} g(n, m) e^{\frac{-2 j u n \pi}{N_{1}}} e^{\frac{-2 j v m \pi}{N_{2}}} \\
& =A_{G}(u, v) e^{j \theta_{G}(u, v)}
\end{aligned}
$$

Where $A_{F}(u, v), A_{G}(u, v)$ are amplitude components and $\theta_{F}(u, v), \theta_{G}(u, v)$ are phase components. The cross phase spectrum is given by

$$
R_{F G}(u, v)=\frac{F(u, v) \overline{G(u, v)}}{|F(u, v) \overline{G(u, v)}|}=e^{j \theta(u, v)}
$$

Where $\overline{G(u, v)}$ is the comlex conjugate of $G(u, v)$ and $\theta(u, v)$ denotes the phase difference $\theta_{F}(u, v)-\theta_{G}(u, v)$. The POC function $r_{f g}(n, m)$ is the $2 \mathrm{D}$ inverse DFT (2D IDFT) of $R_{F G}(u, v)$ given by

$$
r_{f g}(n, m)=\frac{1}{N_{1} N_{2}} \sum_{u=-N}^{N} \sum_{v=-M}^{M} R_{F G}(u, v) e^{\frac{2 j u n \pi}{N_{1}}} e^{\frac{2 j v m \pi}{N_{2}}}
$$

If the matched images $f(n, m)$ and $g(n, m)$ are similar, the POC function gives a distinct sharp peak like Kroneckers delta function $\delta(n, m)$. The hight of the peak gives the similarity measure for image matching. If the images are not similar the peak drops significantly.

\subsection{Spectral Weighting Function}

Since high frequency components have low reliability $\left(\frac{S}{N}\right)$ a spectral weighting function has been used to emphasize these frequencies in order to improve the registration. Gueham et al. 3] proposed a new band-pass-type spectral weighting 
function to enhance recognition rate of shoeprints by eliminating hight frequencies without affecting the peak sharpness given by:

$$
W(u, v)=\left(\frac{u^{2}+v^{2}}{\alpha}\right) e^{-\frac{u^{2}+v^{2}}{2 \beta^{2}}}
$$

Where $\beta$ is parameter for controlling the function width and $\alpha=4 \pi \beta^{4}$ for normalizing the peak between 0 and 1. It has a shape of Laplacian of Gaussian (LoG).The weighting function is applied to cross-phase spectrum, the modified cross-phase spectrum is given by

$$
\widetilde{R}_{F G}(u, v)=\frac{F(u, v) \overline{G(u, v)}}{|F(u, v) \overline{G(u, v)}|} \times W(u, v)=e^{j \theta(u, v)} \times W(u, v)
$$

The modified phase-only correlation (MPOC) is given by

$$
\tilde{r}_{f g}(n, m)=\frac{1}{N_{1} N_{2}} \sum_{u=-N}^{N} \sum_{v=-M}^{M} R_{F G}(u, v) e^{\frac{2 j u n \pi}{N_{1}}} e^{\frac{2 j v m \pi}{N_{2}}} \times W(u, v)
$$

\subsection{Algorithm of Recognition}

Consider an unknown GEI sample from the probe $f_{i}\{i=1 \ldots N\}$, where $N$ is the size of the probe. The algorithm compares this sample to the entire gallery $g_{j}\{j=$ $1 \ldots M\}$ where $M$ is the size of the gallery and determines the matching score between each couple $\left(f_{i}, g_{j}\right)$. The matching score corresponds to the maximum value of the invert Fourier transform of the cross-phase spectrum. After matching an input image from the gallery to all probes we sort the results from the best match to the worst. The input image is identified as the image with the highest score from the gallery. The correct classification rate (CCR) is the ratio of the number of well classified samples over the total number of samples.

\subsection{Supervised Feature Selection}

Feature selection aims to select a subset of relevant features from the initial set. The main goal of supervised feature selection is to enhance the classification accuracy. There exist two families of supervised feature selection: filters and wrappers. The filter approach is independent of the learning algorithm, precedes the classification process and is done once. On the other hand, in a wrapper approach the classification is used itself to measure the importance of features hence this approach achieves better performances since it has a direct interaction with the specific classification method. Due to the large number of possible feature subsets $2^{s}$ (where $s=w \times h, w$ is the width and $h$ is the high of initial GEI) which are usually computationally intensive, a wrapper approach requires a strategy of search to explore efficiently the feature subsets.

To make the strategy of search efficient we have reduces the number of features by considering each row as a feature unit. We have divided the GEI into two 


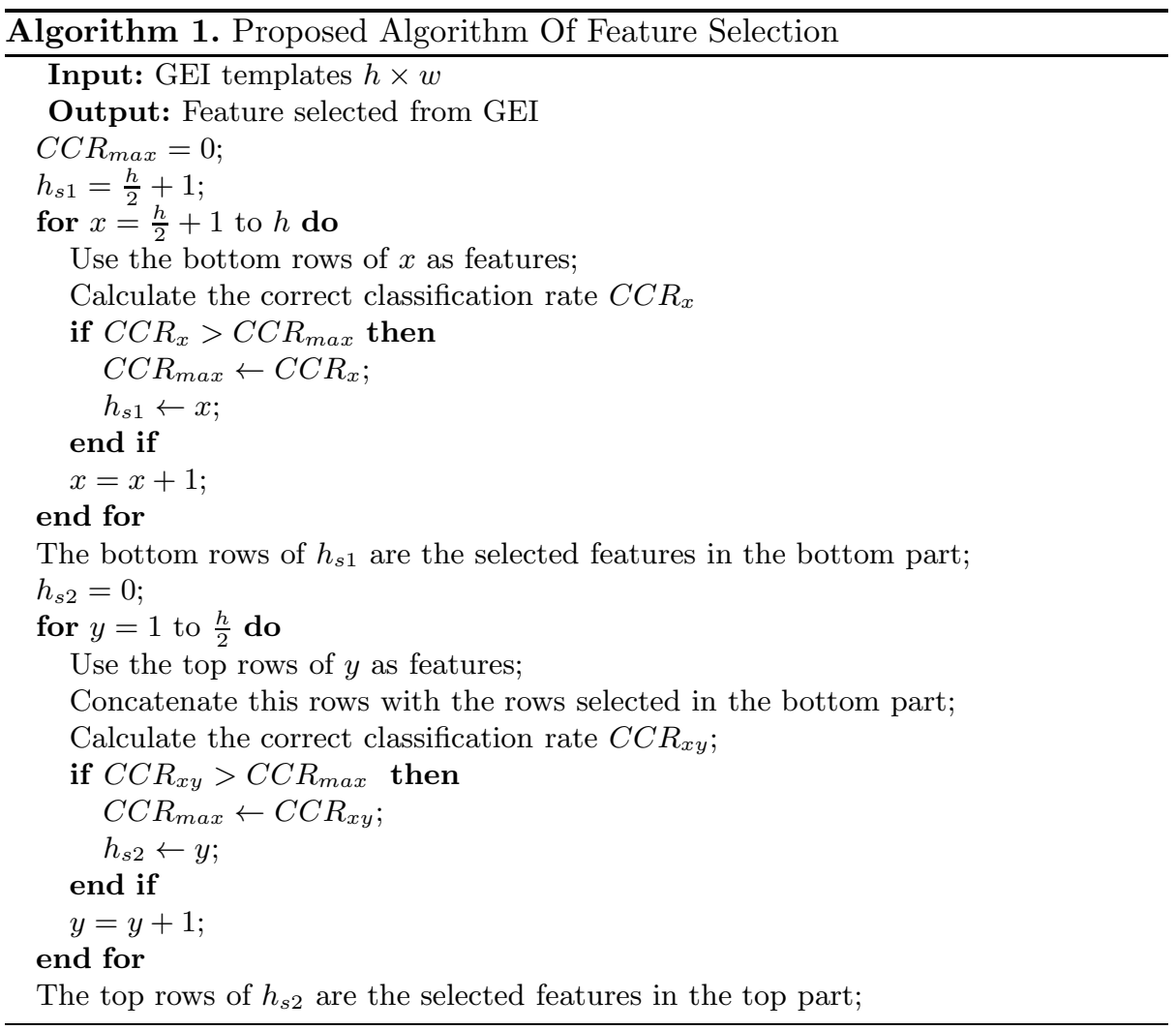

equal parts (top and bottom part). We have removed the rows from the top of the bottom part sequentially (lower part of GEI contains dynamic information which are important for recognition [1]). Once we have found the best feature subset in the bottom part, we have also investigated the top part of the GEI which may contain some informative features (head shape, neck) by adding sequentially rows from top of the top part (see algorithm 1).

\section{Experiments and Results}

We have used CASIA database (dataset B) [10] to evaluate our method. It is a multivew gait dadabase containing 124 subjects captured from 11 different angles starting from $0^{\circ}$ to $180^{\circ}$. Each subject has six normal walking sequences (SetA), two carrying-bag sequences (SetB) and two wearing-coat sequences (SetC). The first four sequences of setA noted as (SetA1) are used for training. The two remaining noted as (SetA2), (SetB) and (SetC) are used for testing the effect of view angle variations, clothing and carrying conditions respectively. In our work we focus on the effect of clothing, carrying conditions and carried out experiments under $90^{\circ}$ view using $64^{*} 64$ GEI resolution. 


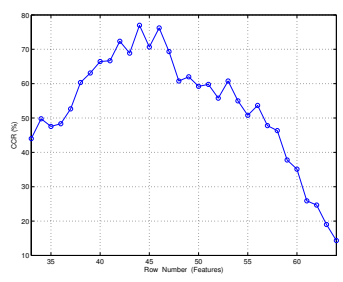

(a) bottom part

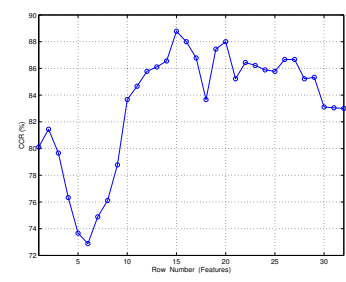

(b) top part

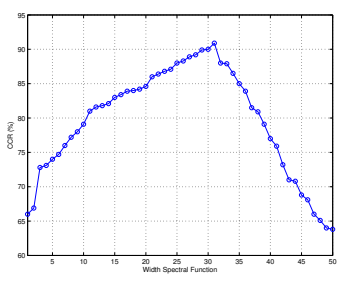

(c) weighting function

Fig. 3. Correct classification rates using various feature subsets and spectral weighting function size

Table 1. Comparison of CCRs (In percent) from several different algorithms on CASIA database using $90^{\circ}$ view

\begin{tabular}{lcccc}
\hline Method & Normal & Carrying-Bag & Wearing-Coat & Mean \\
\hline Bashir et al.[1] & 100.00 & 78.30 & 44.0 & 74.10 \\
Bashir et al.[11] & 97.50 & 83.60 & 48.80 & 76.60 \\
Han et al. [4] & 97.60 & 32.70 & 52.00 & 60.80 \\
Our Method & $\mathbf{9 3 . 6 0}$ & $\mathbf{8 1 . 7 0}$ & $\mathbf{6 8 . 8 0}$ & $\mathbf{8 1 . 4 0}$ \\
\hline
\end{tabular}

We define a feature selection set which should not be specific for a training set, we randomly select with replacement of 24 subjects, for each subject 3 sequences are randomly chosen corresponding to the three variants as consequence we have 72 sequences. We define a 3 -fold cross-validation (SetA1, SetB, SetC), two variants were used for training and the left-out variant for test.

From Fig. 3 it can be seen that rows 44 to 64 in the bottom part, rows 1 to 15 in the top part and the width 31 of the spectral weighting function give the best CCR. Table 1 compares our method against three other existing methods. Our experiments demonstrate that our feature selection improves the recognition performance in the presence of the covariates. The feature selection helps to take in consideration only those features which are not affected by the covariates. Both bottom and top parts of the GEI contribute to discriminate among subjects and we have found in the bottom parts, the dynamic parts of the legs which are already proven that are most informative part. We can also show in our work that the top part of GEI is discriminative and contains parts which help to improve CCR like the head shape and neck as is shown in Fig. 4

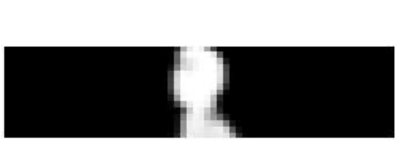

(a) Top part

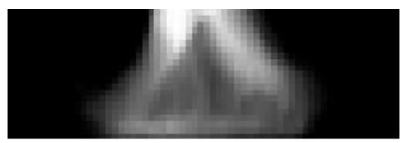

(b) Bottom part

Fig. 4. Feature subset selected in our method 
The quality of the GEI influences significantly the CCR, therfore a good detection, segmentation and tracking are a mandatory to achieve a good performance. Talking about gait recognition, someone can think that is concerning only the moving down parts of the body (legs), but from our work we could notice that also the top parts of the body (head) shape helps to discriminate among people.

\section{Conclusion}

In this paper we have presented a supervised feature extraction method for improved gait recognition. The proposed method demonstrates attractive results especially in presence of covariates and is also computationally effective. As future work we wish to investigate the robustness of our method in the case of view angle variations.

\section{References}

1. Bashir, K., Xiang, T., Gong, S.: Gait recognition without subject cooperation. Pattern Recognition Letters 31(13), 2052-2060 (2010)

2. Foster, J.P., Nixon, M.S., Prugel-Bennett, A.: Automatic gait recognition using area-based metrics. Pattern Recognition Letters 24(14), 2489-2497 (2003)

3. Gueham, M., Bouridane, A., Crookes, D.: Automatic recognition of partial shoeprints based on phase-only correlation. In: IEEE International Conference on Image Processing, ICIP 2007, pp. IV-441-IV-444. IEEE (2007)

4. Han, J., Bhanu, B.: Individual recognition using gait energy image. IEEE Transactions on Pattern Analysis and Machine Intelligence 28(2), 316-322 (2006)

5. Ito, K., Aoki, T., Nakajima, H., et al.: A palmprint recognition algorithm using phase-based image matching. In: 2006 IEEE International Conference on Image Processing, pp. 2669-2672. IEEE (2006)

6. Little, J., Boyd, J.: Describing motion for recognition. In: IEEE Proceedings of the International Symposium on Computer Vision, 1995, pp. 235-240 (1995)

7. Ma, Q., Wang, S., Nie, D., et al.: Recognizing humans based on gait moment image. In: IEEE Eighth ACIS International Conference on Software Engineering, Artificial Intelligence, Networking, and Parallel/Distributed Computing, SNPD 2007, pp. 606-610 (2007)

8. Niyogi, S.A., Adelson, E.H.: Analyzing and recognizing walking figures in XYT. In: Proceedings of the IEEE Computer Society Conference on Computer Vision and Pattern Recognition, CVPR 1994, pp. 469-474. IEEE (1994)

9. Yam, C., Nixon, M.S., Carter, J.N.: Automated person recognition by walking and running via model-based approaches. Pattern Recognition 37(5), 1057-1072 (2004)

10. Yu, S., Tan, D., Tan, T.: A framework for evaluating the effect of view angle, clothing and carrying condition on gait recognition. In: IEEE 18th International Conference on Pattern Recognition, ICPR 2006, pp. 441-444 (2006)

11. Bashir, K., Xiang, T., Gong, S., et al.: Gait Representation Using Flow Fields. In: BMVC, pp. 1-11 (2009) 